\title{
Débit des puits cylindriques pénétrant partiellement la nappe aquifère
}

\author{
Discharge from cylindrical wells \\ penetrating partly into the water table
}

\author{
PAR J. BRILLANT,
}

INGÉNIEUR E.M.P., SOCIÉTĚ 《SOLÉTANCHE》, PARIS

\begin{abstract}
En terrain homogène et dans les conditions de palidité de la loi de Darcy, on démontre que le débit d'un puits pénétrant une nappe captive d'épaisseur $H$ sur une hauteur $L=\lambda H<H$, peut étre évalué en toute rigueur par la formule de Dupuit relative au puits «complet》 $(L=H)$, a condition de prendre en compte un rayon équivalent $R_{E}$ déterminé par la relation:
\end{abstract}

$$
\frac{\mathrm{e} R_{E}}{4 \mathrm{~m} H}=\left(\frac{e R_{\mathrm{p}}}{4 \mathrm{~m} L}\right)^{1 / \lambda} \cdot \Psi(\lambda)
$$

Outre les grandeurs précédemment évoquées, l'équivalence fait intervenir :

- La base des logarithmes népériens $\mathrm{e}=2,718 \ldots$;

- Le rapport $\mathrm{m}^{2}$ - des perméabilités horizontale et verticale;

- Le rayon réel du puits - $R_{\mathrm{p}}$ - considéré comme négligeable;

- Le facteur correctif $\Psi(\lambda)$, fonction connue de $\lambda$, décroissant de: $(\mathrm{e} / \gamma)=1,525$ (pour $\lambda=0$ ) $\dot{a} 1$ (pour $\lambda=1$ ).

L'objet de cette communication est de présenter une nouvelle formule - pour évaluer le débit des puits dits «incomplets » (afin d'exprimer qu'ils n'atteignent pas la limite inférieure, réputée imperméable, de la nappe aquifère).

La formule peut s'imposer par une certaine rigueur; nous donnerons le principe de Ja démonstration après avoir situé le cadre théorique du problème, sans dissimuler certaines difficultés plus ou moins bien résolues, mais sans entrer non plus dans le détail de calculs fastidicux, où l'hydraulique n'est pas spécialement en cause.
It is shown that, given homogeneous soil and conditions in which Darcy's laun applies, the discharge from a well sunk into a water table of depth $H$ to a depth $L=\lambda H<H$ can be rigorously established by Dupuit's formula for a "complete" well $(L=H)$, provided that an equivalent radius $R_{E}$ is taken into considerafion. This radius is determined by the relation:

$$
\frac{e R_{k}}{4 \mathrm{~m} H}=\left(\frac{\mathrm{e} R_{\mathrm{p}}}{4 \mathrm{~m} L}\right)^{1 / \lambda} \cdot *(\lambda)
$$

In addition to the above values, the equivalence involves the following:

- The natural logarithm base e $=2.718 \ldots$.

- The horizontal/pertical permeability ratio $\mathrm{m}^{2}$;

- The true radius of the well $R_{\mathrm{p}}$, taken as negligible;

The correction factor $\Psi(\lambda)$, which is a known function of $\lambda$, diminishing from $\mathrm{e} / \gamma=1.525$ (where $\lambda=0$ ) to 1 (where $\lambda=1)$.

Nous espérons ainsi pouvoir insister sur des notions plus propres à l'hydrologie souterraine (surfaces libres et anisotropie des terrains alluvionnaires), en tenir compte sans compliquer les calculs, et confronter sur un exemple quelques formules existantes.

$$
* *
$$

Nous nous placons dans le cadre le plus strict de la loi de Darcy où le milieu poreux, homogène et isotrope est entièrement défini par sa perméabilité à l'eau, $K$, évaluée par une «vi- 


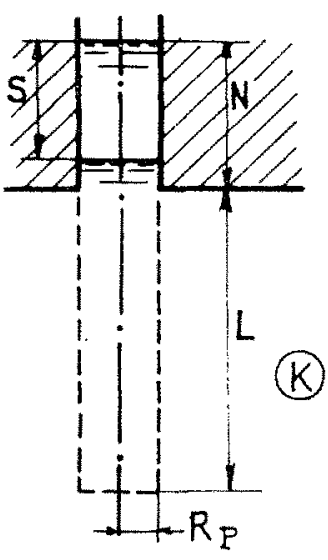

FIG. $1 t$

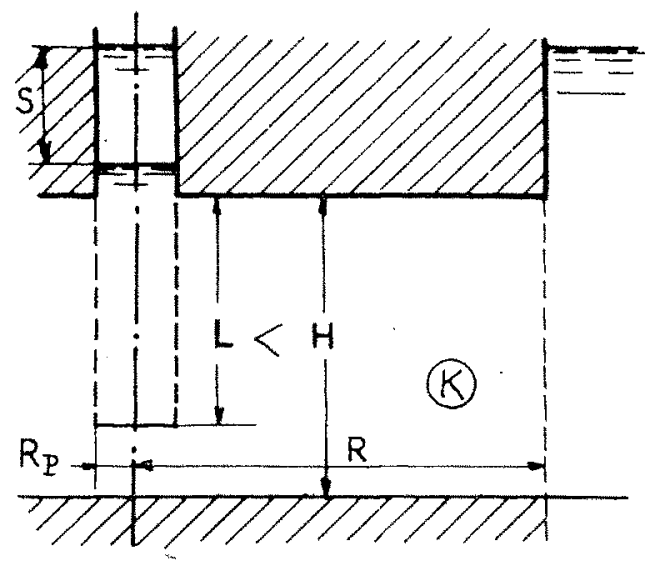

Fia. $1 b$

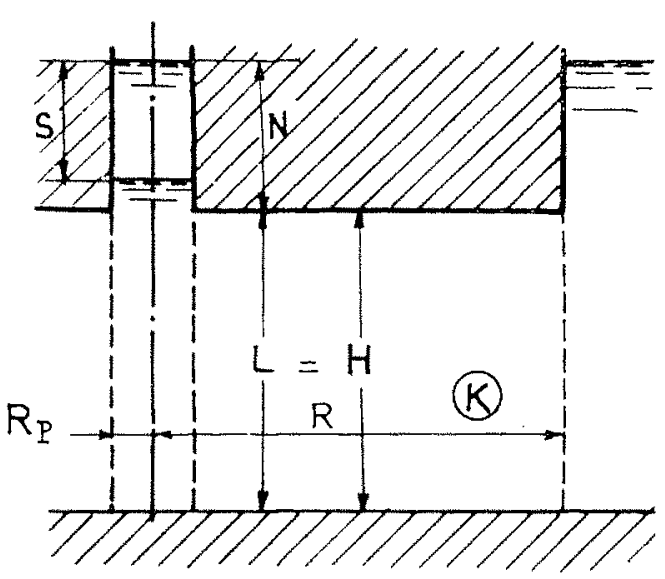

FIG. $1 c$ tesse $\gg$, et nous considérons (fig. $1 a, 1 b$, et $1 c$ ) une nappe captive drainée par un puits cylindrique vertical de hauteur $\mathrm{L}$ et rayon $\mathrm{R}_{p}$ où le rabattement $S$ ne dépasse pas la mise en charge $N$ de la nappe; on dispose de deux formules classiques :

-. La formule de l'ellipsoïde en l'absence de substratum (fig. 1 a):

$$
\mathrm{Q}_{p}>\mathrm{Q}=\frac{2 \pi \cdot \mathrm{KLS}}{\ln 2 \mathrm{~L} / \mathrm{R}_{p}}
$$

dans laquelle le débit du puits - $\mathrm{Q}_{p}$ - est sousestimé par le débit $Q$ correspondant au même puits limité au demi-ellipsöde inscrit, de faible rayon $\left(\mathbf{R}_{p}\right)$ devant la longueur $(L)$ : il s'agit plus précisément d'un cas limite $\left(\mathrm{R}_{p} / \mathrm{L} \rightarrow 0\right)$ où $Q$ aussi bien que $Q_{p}$ tendent vers zéro, mais sont « équivalents» [avec $\left.\left(Q_{p} / Q\right) \rightarrow 1\right]$.

- L'autre est la formule de Dupuit (fig. $1 \mathrm{c}$ ), rigoureuse, pour un puits atteignant le substratum à la profondeur $\mathrm{H}(=\mathrm{L})$ :

$$
\mathrm{Q}_{p}=\frac{2 \pi \cdot \mathrm{KHS}}{\ln \mathrm{R} / \mathrm{R}_{p}}
$$

où l'évaluation de $Q_{p}$ est subordonnée à l'introduction d'une donnée supplémentaire, le « rayon d'action » $R$, fini mais grand par rapport $\dot{a} \mathbf{H}$, par lequel on admet que tout se passe comme si la nappe était alimentée sans perte de charge par une réserve à niveau fixe, baignant circulairement à cette distance toute l'épaisseur de l'aquifère.

$$
\text { * }
$$

En première étape, on reprend la formule de l'ellipsoïde dont le rabattement est identifié (fig. 2) à celui provoqué par le segment $2 \mathrm{~L}$ (de part et d'autre de l'horizontale représentant le «toit» et l'origine des ordonnées), segment uniformément drainé par le débit $2 \%$, c'est-àdire par une «répartition» constante

$$
q_{0}=(\mathrm{Q} / \mathrm{L})
$$

et ce rabattement évalué en $A$, à la distance $R_{p}$, par la somme des influences élémentaires :

$$
\frac{d \mathrm{Q}\left(=q_{0} d z\right)}{4 \pi \mathrm{K}_{?}}
$$

o étant la distance des éléments $d z$ au point $A$ (sur l'ellipsoïde) où l'on calcule le rabattement.

En présence d'un substratum B, le rabattement au même point peut être évalué en ajoutant l'influence de toutes les images successives du segment initial par rapport au «miroir » $B$ et son symétrique $\mathrm{B}^{\prime}$. Mais le rabattement en $\mathrm{A}$, dont chaque terme correspond à l'influence d'un couple d'images d'indice $n$, se présente alor's sous la forme d'une série de terme général d'ordre $[1 / n(\rightarrow \infty)]$, done divergente.

Pour obtenir un rabattement fini, on est amené à tenir compte de la réalimentation à distance finie, dont l'influence sera identifiée à celle du débit $(-2$ Q) uniformément réparti sur chaque surface cylindrique de rayon $R_{a}$ et hauteur $2 \mathrm{H}$, ou, ce qui revient au même, sur chaque segment de génératrice tel que $\mathrm{GG}^{\prime}$. Leur ensemble donne lieu à une deuxiène série en $1 / n$ dont la différence terme à terme avec la première est une série convergente (en $1 / n^{2}$ ). Sa somme peut être évaluée sans difficulté en faisant intervenir la fonction «factorielle» généralisée pour $x$ quelconque (non entier) et il vient, tous calculs faits, une formule analogue à la formule de Dupuit :

$$
Q=\frac{2 \pi \cdot \mathrm{KHS}}{\ln \mathrm{R}_{a} / \mathrm{X}_{n}}
$$

avee :

$$
\mathrm{X}_{0}=4 \mathrm{H}\left[\frac{\mathrm{R}_{p}}{2 \mathrm{~L}} \cdot \frac{(\mathrm{L} / 2 \mathrm{H}) !}{(-\mathrm{L} / 2 \mathrm{H}) !}\right]^{\mathrm{H} / \mathrm{L}}
$$




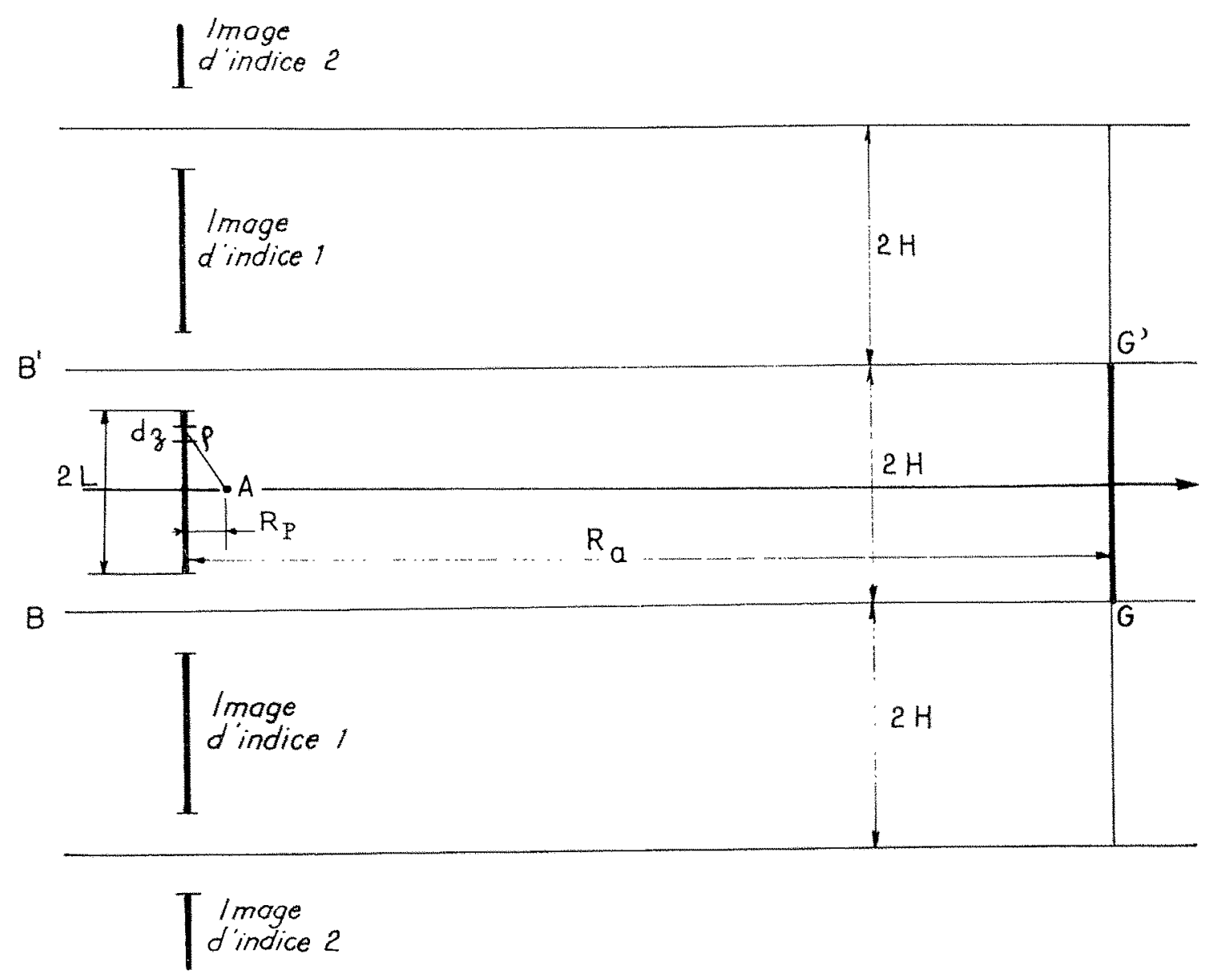

FIG. 2

ou, $\mathrm{X}_{0}$ défini par :

$$
\frac{\mathrm{X}_{0}}{2 \mathrm{H}}=\left(\frac{\mathrm{R}_{p}}{2 \mathrm{I}}\right)^{\mathrm{H} / \mathrm{L}} \times \Phi\left(\frac{\mathrm{L}}{\mathrm{H}}\right)
$$

avec :

$$
\Phi\left(\frac{\mathrm{L}}{\mathrm{H}}\right)=2\left[\frac{(\mathrm{L} / 2 \mathrm{H}) !}{(-\mathrm{L} / 2 \mathrm{H}) !}\right]^{\mathrm{T} / \mathrm{L}}
$$

Pour $\mathrm{L}=\mathrm{H}$ :

$$
\Phi\left(\frac{\mathrm{L}}{\mathrm{H}}\right)=1, \quad \mathrm{X}_{0}=\mathrm{R}_{p}
$$

et on retrouve la formule de Dupuit :

$$
\mathrm{Q}=\frac{2 \pi \cdot K H S}{\ln \mathrm{R}_{i} / \mathrm{R}_{p}}
$$

$Q$ étant alors rigoureusement égal à $Q_{p}$, on en déduit :

$1^{\circ}$ que la distance prise en compte pour la réalimentation $\left(R_{0}\right)$ doit être identifiée au " rayon d'action» $R$ de la formule de Dupuit;

$2^{\circ}$ qu'on obtient dans le cas général (fig. $1 b$, $L<H)$ une approximation sur le débit au moins égale à celle de l'ellipsoïde inscrit à l'égard du puits cylindrique, en substituant $X_{0}$ à $R_{p} \ldots$ Mais dans le cadre de cette approximation, il semble qu'il n'y ait pas lieu de conserver le facteur égal à 1 pour $\mathrm{L} / \mathrm{H}=1$ dont le maximum (pour $\mathrm{L} / \mathrm{H}=0$ ) est limité à $2 / \gamma=1,123 . \bar{j} \not 1$.

D'ailleurs, si l'on ne se préoccupe que de l'équivalence entre $Q_{p}$ (débit réel) et $Q$ (débit évalué) à la limite $\left(R_{p} / L\right) \rightarrow 0$, toute substitution $\alpha \mathrm{X}_{0}$ (pour $\alpha$ fini) est tout aussi valable sans que l'une puisse être plus précisément reconnue comme «rayon équivalent» à substituer à $\mathbf{R}_{p}$ dans le cas limite. On peut supposer que c'est à cause de cette indétermination, plus apparente que réelle en ce qui concerne l'approximation du débit, que la formule établie sans grande difficulté et par un processus assez naturel, n'a pas encore été proposée... à notre connaissance.

\section{*3:}

Mais nous allons pouvoir préciser le rayon équivalent : dans le cas d'une répartition linéaire $q_{0}=(\mathrm{Q} / \mathrm{L})$ (isolée sur un seul segment $2 \mathrm{~L}$ pour 
fixer les idées), on peut calculer à la distance $\mathrm{R}_{p}$ et à l'ordonnée $z$ le rabattement (précédemment évalué à l'ordonnée zéro) sous la forme :

$$
\mathrm{S} q_{0}=\frac{q_{0}}{2 \pi \mathrm{K}}\left[\ln \frac{2 \mathrm{~L}}{\mathrm{R}_{p}}+\ln \sqrt{\mathrm{1}-\mathrm{z}^{2} / \mathrm{L}^{2}}\right]
$$

On distingue ainsi :

un terme fixe infini (pour $\left(\mathrm{R}_{p} / \mathrm{L}\right) \rightarrow 0$ :

$$
\frac{q_{0}}{2 \pi \mathrm{K}} \ln \frac{2 \mathrm{~L}}{\mathrm{R}_{p}}
$$

une fonction finie de $z$ :

$$
\frac{q_{0}}{2 \pi \mathrm{K}} \ln \sqrt{1-z^{2} / \mathrm{L}^{2}}
$$

et l'idée vient assez naturellement que la «partie principale infinie » du rabattement au niveau $z$ peut être liée de manière plus générale à la valeur prise au même niveau par une répartition $q$ fonction de $z$. On démontre effectivement que le rabattement sous l'influence de $q(z)$ est de la forme :

$$
\begin{aligned}
\mathrm{S} q=\frac{q(z)}{2 \pi \mathrm{K}}[ & \ln \frac{2 \mathrm{~L}}{\mathrm{R}_{p}} \\
& + \text { (fonctionnelle finie de } q \Rightarrow \varphi(z)]
\end{aligned}
$$

Avec la distribution :

$$
q=q_{0}-\frac{q_{0} \ln \sqrt{1-z^{2} / L^{2}}}{\ln 2 \mathrm{~L} / \mathrm{R}_{p}}
$$

On aura done :

$$
\begin{array}{r}
\mathrm{S} q=\frac{q_{0}}{2 \pi \mathrm{K}}\left[\ln \frac{2 \mathrm{~L}}{\mathrm{R}_{p}}+\ln \sqrt{1-\frac{z^{2}}{\mathrm{~L}^{2}}}\right] \\
-\frac{q_{0} \ln \sqrt{1-\left(z^{2} / \mathrm{L}^{2}\right)}}{2 \pi \mathrm{K} \ln \left(2 \mathrm{~L} / \mathrm{R}_{p}\right)}\left[\ln \frac{2 \mathrm{~L}}{\mathrm{R}_{p}}+\vartheta^{(z)}\right] \\
=\frac{q_{0}}{2 \pi \mathrm{K}}\left[\ln \frac{2 \mathrm{~L}}{\mathrm{R}_{p}}+\varepsilon(z)\right]
\end{array}
$$

La distribution considérée - en retranchant à la répartition constante $q_{0}$ une répartition infiniment petite, proportionnelle à l'accroissement, entre 0 et $z$, du rabattement provoqué par $q_{0}-$, nous donne donc à la distance $R_{p}$ (c'est-á-dire sur la surface cylindrique du puits) un rabattement infini constant $\dot{a}$ un infiniment petit près, au lieu du rabattement infini constant $\dot{a}$ une fonction finie près pour la répartition $q_{0}$; le même principe appliqué au cas «avec substratum», on obtient successivement : - le débit du puits en nappe semi-indéfinie :

$$
\mathrm{Q}_{p}=\frac{2 \pi \mathrm{KLS}}{\ln \frac{2 \mathrm{~L}}{\mathrm{R}_{p}(e / 2)\left(1+\varepsilon_{n}\right)}}(e=2,718 \ldots)
$$

c'est-à-dire (à la limite $\varepsilon_{p} \rightarrow 0$ avec $\left.\frac{\mathrm{R}_{p}}{\mathrm{~L}} \rightarrow 0\right)$, le débit correspondant non plus à l'ellipsoïde inscrit (de rayon $\mathbf{R}_{p}$ ), mais à l'ellipsoide de même hauteur et rayon $(e / 2) \mathrm{R}_{p}$ (fig. 3 ); ce "rayon équivalent d'ellipsoïde " est d'ailleurs aussi bien valable pour un puits à fond crépiné qu'à fond plein, la distinction ne devant apparaître que dans l'expression de l'infiniment petit $\varepsilon_{p}$

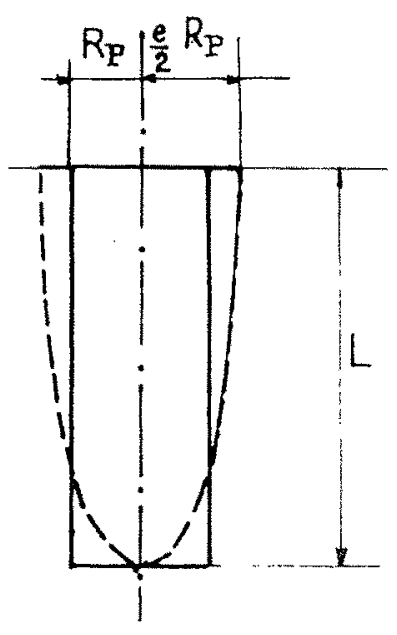

Fig̣. 3

- en présence d'un substratum, une valeur limite $\left[\right.$ pour $\left(R_{p} / \mathrm{L}\right) \rightarrow 0$ ] du rayon à substituer dans la formule de Dupuit, rayon $R_{\mathrm{F}}$ défini par :

$$
\frac{e \mathrm{R}_{\mathrm{I}}}{4 \mathrm{H}}=\left[\frac{e \mathrm{R}_{p}}{4 \mathrm{~L}}\right]^{\mathrm{H} / \mathrm{L}} \mathrm{\Psi}\left(\frac{\mathrm{L}}{\mathrm{H}}\right)
$$

L'expression de $\Psi(\mathrm{L} / \mathrm{H})$ est assez compliquée; mais compte tenu de ses limites:

un maximum $(e / \gamma)=1,525$ pour $(\mathrm{L} / \mathrm{H})=0$, un minimum égal à 1 , avec tangente verticale, pour $(\mathrm{L} / \mathrm{H})=1$ le calcul de troís points nous a paru suffisant pour déterminer $\Psi$, admettre l'ajustement elliptique

$$
\log \Psi=0,184 \sqrt{1-\mathrm{L}^{2} / \mathrm{H}^{2}}
$$

et en définitive, $R_{\mathrm{E}}$ défini par :

$$
\frac{e \mathbf{R}_{\mathrm{L}}}{4 \mathrm{H}}=\left[\frac{e \mathrm{R}_{y}}{4 \mathrm{~L}}\right]^{\mathrm{H} / \mathrm{L}} \cdot 10^{0,184} \sqrt{1=1 / \mathrm{H}^{3}}
$$

Ici, nous arrivons aux « difficultés mal résolues $»$ : que devient le facteur $\Psi$, ou si l'on préfère la courbe d'équation :

$$
\mathrm{Y}=0,184 \sqrt{\mathbf{1}-\mathrm{L}^{2} / \mathrm{H}^{2}}
$$

ajustée à $\ll \log \Psi »$, pour des valeurs pratiques, petites mais non nulles, de $\mathrm{R}_{p} / \mathrm{L}$ ? L'analyse ne 


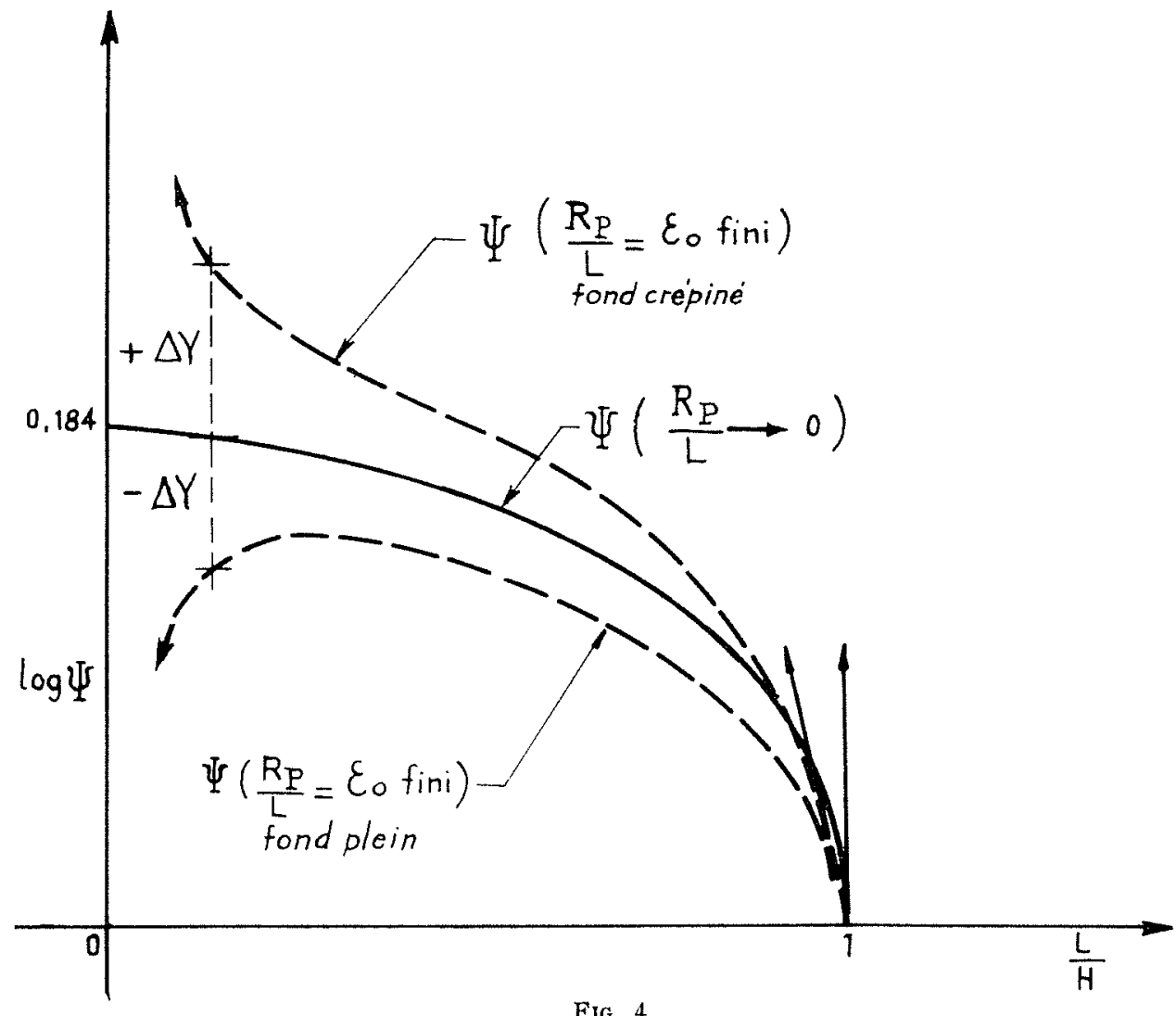

FIG. 4

nous a pas permis de préciser grand chose à ce sujet sinon que :

$1^{\circ} \mathrm{Au}$ voisinage de l'origine (fig. 4), pour $(L / H) \rightarrow 0$ ) le supplément d'ordonnée $\Delta \mathrm{Y}$ est lié à $\varepsilon_{p}$ par :

$$
\Delta \mathrm{Y}=\frac{\mathrm{H}}{\mathrm{L}} \log \left(1+\varepsilon_{p}\right) \# \frac{\varepsilon_{p}}{2,3} \frac{\mathrm{H}}{\mathrm{L}}
$$

et devient done infini sauf pour $s_{p}$ rigoureusement nul, ce qui ne semble pas le cas (et de toute manière certainement pas à la fois pour les puits à fond plein ou crépiné). En fait, il y a une présomption, confirmée par «modèle analogique », que $\Delta Y$ soit positif avec fond crépiné, négatif avec fond plein et que la courbe $\log \Psi$ sépare dans cette zone le domaine des deux «catégories» de puits.

$2^{\circ}$ Au contraire, au voisinage de $(\mathrm{L} / \mathrm{H})=1$ (fig. 4), les courbes relatives à $\left(R_{p} / L\right) \neq 0$ doivent admettre des tangentes non verticales et passer toutes au-dessous de la courbe $\mathrm{Y}=\log \Psi$ à tangente verticale : sinon le rayon équivalent d'un puits «presque complet» pourrait devenir légèrement supéricur à celui du puits complet.

Pour essayer d'en savoir un peu plus, nous avons fait appel à l'analogie électrique; le mon-

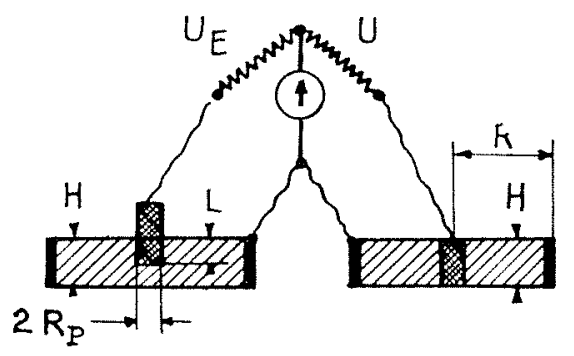

Fig. 5

tage est relativement simple (fig. 5). Les résistances entre électrodes-puits et les parois latérales métalliques de deux «cuves» de rayon $R$ sont dans Je rapport:

$$
\frac{\log \mathrm{R}-\log \mathrm{R}_{n}}{\log \mathrm{R}-\log \mathrm{R}_{\mathrm{K}}}
$$

mesuré à l'équilibre d'un pont de Wheatstone, par le rapport connu $\mathrm{M}$ des résistances $\mathrm{U}$ et $\mathrm{U}_{\mathrm{J}}$. Ón en déduit $R_{\mathrm{E}}$ puis :

$$
\frac{e \mathrm{R}_{\mathrm{I}} / 4 \mathrm{H}}{\left(e \mathrm{R}_{p} / 4 \mathrm{~L}\right)^{\mathrm{H} / \mathrm{L}}}=\Psi \text { (expérimental). }
$$

On constate sur la figure 6 que les différentes «formes» de points correspondant à des valeurs 


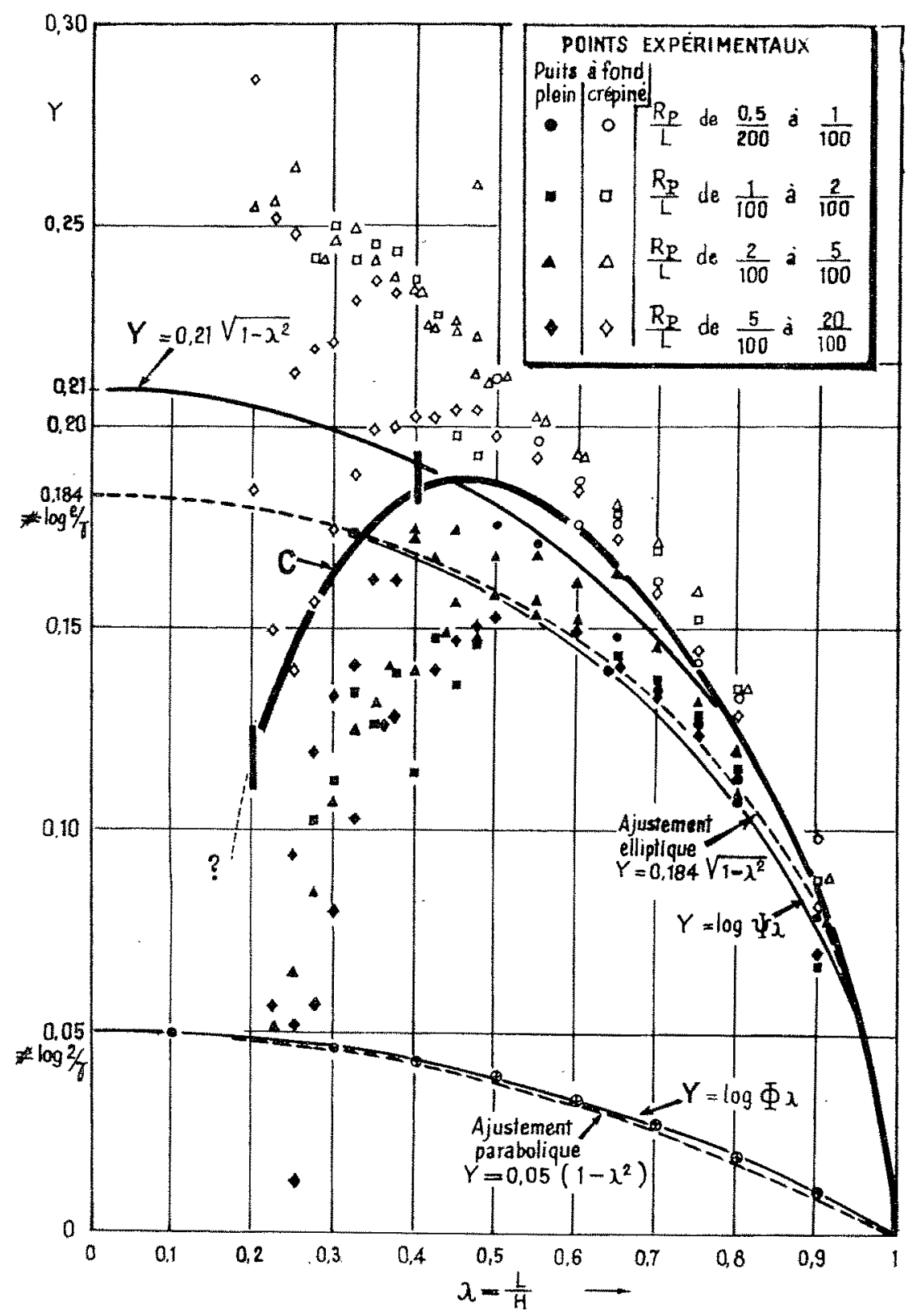

Frg. 6

de $R_{p} / L_{\text {}}$ échelonnées de 0,5 à 20 centièmes $(=0,2)$ ne donment pas lieu à un «classement» significatif; par contre la séparation des «points blancs» et des «points noirs» pour les puits à fond crépiné ou non est assez nette et la courbe séparatrice $\mathrm{C}$ peut donc être considérée comme leur limite commune pour $\left(R_{p} / L\right) \rightarrow 0$, c'est-adire comme une approximation expérimentale de la courbe $\log$ Y qui ne semble pas trop mauvaise (vis-à-vis de la courbe théorique), sur la partie droile du graphique $[(\mathrm{L} / \mathrm{H})>0,4]$. En deçà $[(L / H)<0,4]$, la courbe $C$ paraît s'infléchir vers le bas, sans doute à cause des résistances de contact prépondérantes sur les électrodes trop courtes.

Nous retiendrons surtout que les écarts expérimentaux avec la courbe $C$ représentent plus ou moins grossièrement les écarts théoriques avec la courbe $\log \Psi$, pour des valeurs $\left(R_{p} / L\right) \leqslant 0,2$ et atteignent environ :

$$
0,04 \text { pour }(\mathrm{L} / \mathrm{H})=0,4
$$

et :

0,1 pour $(\mathbf{L} / \mathbf{H})=0,2$ 
En conclusion, nous majorons le facteur $0,184(\log e / \gamma)$ du terme :

$$
\frac{1}{10} \frac{R_{p} H}{L^{2}}\left(=\Delta Y, \text { pour } \frac{L}{H}=0 \text { et } \sqrt{1-L^{2} / H^{2}}=1\right)
$$

(avec le signe + pour les puits à fond crépiné et - - pour le fond plein) terme qui correspond effectivement

- à lordre de grandeur des écarts observés

$$
\text { pour } \frac{\mathrm{R}_{n}}{\mathrm{~L}}<0,2 \text { et } \frac{\mathrm{L}}{\mathrm{H}}=0,4 \text { ou } 0,2
$$

- et à une expression de $\varepsilon_{p}$ :

$$
\varepsilon_{p}= \pm 0,23 \frac{\mathrm{R}_{p}}{\mathrm{~L}}
$$

du premier degré en $R_{p} / L$, done assez « naturelle $\gg$.

En définitive, nous proposons la formule conplète :

$$
\frac{e \mathbf{R}_{\mathrm{E}}}{4 \mathrm{H}}=\left[\frac{e \mathrm{R}_{p}}{4 \mathrm{~L}}\right]^{\mathrm{H} / \mathrm{L}} \cdot 10^{\left[0,184 \pm\left(\mathrm{I}_{p} \mathrm{H} / 10 \mathrm{~L}^{9}\right)\right]} \sqrt{1-\mathrm{L}^{3} / \mathrm{H}^{2}}
$$

mais nous limitons le terme correctif

$$
\left(\mathrm{R}_{p} \mathrm{H} / 10 \mathrm{~L}^{2}\right)
$$

à 0,2 ce qui s'exprime par une condition d'application peu restrictive :

$$
\frac{\mathrm{R}_{n}}{\mathrm{~L}}<2 \frac{\mathrm{L}}{\mathrm{H}}
$$

Enfin, si l'on tient absolument à éviter tout ecart possible dans le mauvais sens $»\left(R_{\mathrm{E}}>\mathrm{R}_{p}\right)$ pour des puits «presque complets», il faut exclure les cas où $(H-L) / H$ est inférieur à $0,5 /\left(\ln ^{2} \mathrm{H} / 2 \mathrm{R}_{p}\right)$.

\section{$* *$}

\section{TFRRAINS ANISOTROPES :}

Les terrains alluvionnaires sont normalement doués d'une «anisotropie de révolution verticale » résultant des conditions d'alluvionnement (dépôt «à plat» de particules non sphériques, mais surtout «cycles d'alluvionnement» superposant des horizons de perméabilités différentes), anisotropie caractérisée par le rapport $\left(\mathrm{K}_{h} / \mathrm{K}_{v}\right)=m^{2}$ des perméabilités horizontale et verticale :

On sait qu'un tel milieu peut être identifié au milieu isotrope de perméabilité $K_{h}$ dont les dimensions horizontales sont divisées par

$$
m=\sqrt{\left(\mathrm{K}_{h} / \mathbf{K}_{v}\right)}
$$

On vérifie dans ces conditions que la formule de Dupuit reste valable à condition de considérer K comme la perméabilité horizontale; par contre, la formule qui définit le rayon équivalent devient :

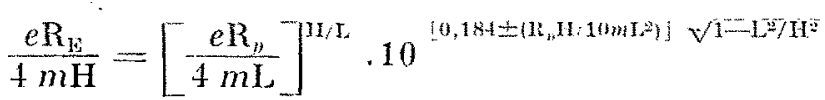

Le rapport $m^{2}$ est couramment évaluć entre 5 et 10 ; en labsence de donnée précise à cel égard, on peut donc accepter une valeur moyenne $m^{2}=\sqrt{5.10}$ et pour simplifier la formule, assimiler (ici comme par la suite)

$$
m=\sqrt[4]{50}=2,66 \text { à } e=2,71 \ldots
$$

Mais dans le cadre de cette indétermination, le facteur $10^{(0,181 \ldots)}$ [compris entre 0,95 et 2,5 compte tenu de $\left.(1 / 10)\left(\mathrm{R}_{p} \mathrm{H} / \mathrm{mL}^{2}\right)<0,2\right]$ devient illusoire, et il suffit de retenir :

$$
\frac{\mathrm{R}_{\mathrm{E}}}{4 \mathrm{H}}=\left[\frac{\mathrm{R}_{p}}{4 \mathrm{~L}}\right]^{\mathrm{H} / \mathrm{L}}
$$

grossièrement valable pour tout puits allongé $\left(\mathrm{L},>2 \mathrm{R}_{p}\right)$.

$$
* *
$$

RABATTEMENT DANS L'AQUIFERF ( $\left.\mathbf{S}^{\prime}\right)$. - Lorsque le puits complet est rabattu d'une hauteur $S^{\prime}$ par rapport à une surface libre ou que son rabattement dépasse de $\mathrm{S}^{\prime}=\mathrm{S}-\mathrm{N}$ la mise en charge de la nappe captive, on dispose d'une autre «formule de Dupuit», démontrée en toute rigueur par Tcharnyi [6], pour évaluer le débit; arec nos notations:

$$
\mathrm{Q}_{n}=\pi \mathrm{K} \frac{2 \mathrm{H}\left(\mathrm{N}+\mathrm{S}^{\prime}\right)-\mathrm{S}^{2}}{\ln \mathrm{R} / \mathrm{R}_{n}}
$$

$\left(\mathrm{N}=0\right.$ d'où $\mathrm{S}^{\prime}=\mathrm{S}$ pour une nappe libre).

Mais, dans ces conditions, rien ne permet d'affirmer que le rayon équivalent $R_{E} d u$ puits incomplet, précédemment évalué pour une nappe captive sans rabattement dans l'aquifère $(S<N)$ en fonction des seules domnées $\mathrm{H}, \mathrm{L}$ et $\mathrm{R}_{p}$ (ou $\left.\mathrm{R}_{p} / m\right) \ldots$ soit encore valable en remplacement du rayon réel $\mathrm{R}_{n}$; il semble en fait qu'il $\mathrm{y}$ ait alors lieu d'évaluer un rayon équivalent difrérent --nous l'appelons $R_{E}^{\prime}$ pour éviter loute confusion - fonction décroissante de $S^{\prime}$ entre autros.

Inversement, tonte formule générale concernant le débit d'un puils incomplet, avec ou sans rabattement dans l'aquifère, permet de faire apparaitre un ravon équivalent $R_{E}$ ou $R_{E}^{\prime}$ : il sumft, en effet, d'éliminer $\mathrm{Q}_{p} / \mathrm{K}$ entre cette formule et la formule de Dupuit correspondante. 
Mais $R^{\prime}{ }_{E} \rightarrow R_{E}$ et toutes autres données que $H$, $\mathrm{L}$ et $\mathrm{R}_{p}$ (ou $\mathrm{R}_{p} / \mathrm{m}$ ) doivent s'éliminer, le rayon d'action $R$ en particulier à la limite $S^{\prime} \rightarrow 0$ (où les «deux formules de Dupuit» se confondent).

Même sans faire état des bases théoriques qui justifient I'expression de $R_{\mathrm{E}}$ et font l'objet principal de cette communication, aucune des formules existant à notre connaissance, ne satisfait à l'ensemble de ces conditions.

D'une part MM. Li, Benton et Bock [3] semblent être les seuls à avoir proposé pour une nappe captive (et $S<N$ ) une expression de $R_{E}$ indépendante de $R$ (pour un puits à fond plein) :

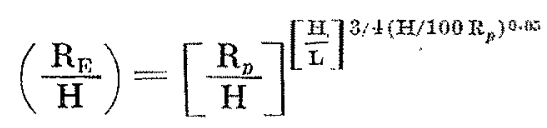

mais sans préciser, à notre connaissance, s'ils envisagent la même substitution $\left(R_{E}\right)$ ou celle d'un rayon plus ou moins réduit $\left(R_{\mathrm{E}}\right)$ dans la formule de Dupuit rèlative au cas où le rabattement intéresse l'aquifère.

D'autre part, les résultats de M. Boreli [4] paraissent indiscutables en ce qui concerne sept cas particuliers « en nappe libre» résolus par relaxation.

Les six premiers nous ont d'ailleurs permis de vérifier un ajustement excellent de notre formule en évaluant $R_{E}^{\prime}$ comme $R_{E}$ après avoir simplement réduit la longueur $\mathrm{L}$ de la quantité :

$$
4 \mathrm{~S}^{\prime 2}(\mathrm{H}-\mathrm{L})^{2} / \mathrm{H}^{3}
$$

qui s’annule comme il se doit pour

$$
\mathrm{S}^{\prime}=0 \text { ou } \mathrm{H}-\mathrm{L}=0
$$

par contre, comme celles de Forchheimer [1] et Kozeny [2], la formule que propose $M$. Boreli fait apparaître un rayon équivalent, défini avec nos notations par :

$$
\frac{\mathrm{R}_{\mathrm{E}}}{\mathrm{R}}=\left[\frac{\mathrm{R}_{p}}{\mathrm{R}}\right]^{\frac{\left(\mathrm{II}-\mathrm{S}^{\prime}\right) /\left(\mathrm{L}-\mathrm{S}^{\prime}\right)}{1+\left(0,20+10 \mathrm{R}_{p} / \mathrm{L}\right) \sin 1,8[(\mathrm{~K}-\mathrm{L}) / \mathrm{H}]}}
$$

qui dépend encore du rayon d'action $R$ à la limite $S^{\prime} \rightarrow 0$.

$$
\stackrel{*}{*}
$$

Nous confrontons le résultat de différentes formules dans un cas simple, sans rabattement dans l'aquifère $(\mathrm{S}<\mathrm{N})$ avec :

$$
\mathrm{H}=10 \mathrm{~m}, \quad \mathrm{~L}=5 \mathrm{~m}, \quad \mathrm{R}=200 \mathrm{~m}
$$

et

$$
\mathrm{R}_{p}=200 \mathrm{~mm}
$$

(d'où $\mathrm{L} / \mathrm{H}=1 / 2$ et $\mathrm{R}_{p} / \mathrm{L}_{\text {, }}=1 / 25$ ).

Nous avons choisi à dessein un cas où notre résultat, (avec fond plein) est très peu différent de celui de MM. Li, Benton et Bock. En effet, la comparaison facile de formules qui font intervenir les mêmes données $\left(H, L\right.$ et $\left.R_{p}\right)$ montre que l'évaluation ancienne de $R_{\mathbb{K}}$ sera par défaut avec

$$
\frac{\mathrm{L}}{\mathrm{H}}<\frac{1}{2} \text { et } \frac{\mathrm{R}_{p}}{\mathrm{~L}}<\frac{1}{25} \text {, }
$$

par excès dans le cas contraire. On note d'ailleurs la faible influence que « l'expérience analogique » reconnait au fond (crépiné ou non) pour un rapport $\left(\mathbf{R}_{p} / \mathbf{L}\right)=1 / 25$ qui n'est pas tellement négligeable.

\begin{tabular}{|c|c|c|c|}
\hline & Fond & $\begin{array}{l}\mathrm{R}_{\mathrm{a}} \\
(\mathrm{mm})\end{array}$ & $\begin{array}{c}\mathrm{Q}_{p} / \mathrm{Q}_{0} \\
\text { (puits complet } \\
\text { de même rayon) }\end{array}$ \\
\hline Forchheimer $(1898), \ldots \ldots \ldots \ldots \ldots \ldots \ldots$ & non précisé & 29,36 & 0,783 \\
\hline 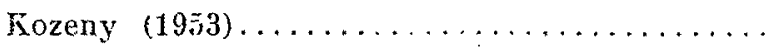 & crépiné & 59,90 & 0,850 \\
\hline Li, Benton et Bock $(1954) \ldots \ldots \ldots \ldots \ldots \ldots$ & plein & 15,58 & 0,715 \\
\hline 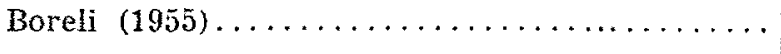 & crépiné & 7,69 & 0,679 \\
\hline \multirow{2}{*}{ Formule actuelle $\ldots \ldots \ldots \ldots \ldots \ldots \ldots \ldots \ldots \ldots$} & crépiné & 15,98 & 0,732 \\
\hline & plein & 15,75 & 0,728 \\
\hline
\end{tabular}

On notera également l'évaluation par défaut de M. Boreli; comme nous admettons le résultat de ses cas de référence, l'écart peut provenir d'un mauvais ajustement de sa formule à la limite $\mathrm{S}^{\prime} \rightarrow 0$, mais surtout du rayon d'action, qui intervient ici en facteur d'exposant négalif, et qui est beaucoup plus grand dans notre 
exemple $(20 \mathrm{H})$ que celui pris en compte dans les modèles de référence $(0,5$ à $2 \mathrm{H})$.

\section{* *}

Pour terminer, nous rappelons que les rayons équivalents mis en cause, constituent un moyen de calcul, pas toujours une «réalité physique», si l'on tient compte des limites d'application de la loi de Darcy [5], sommairement exprimées par une vitesse limite sous les forts gradients.

A ce point de vue, il ne viendrait certes à l'idée de personne de conlondre un puits de dimensions raisonnables $-1 \mathrm{~m}$ de diamètre et pénétrant de quelque 2 à $3 \mathrm{~m}$ une formation aquifère de $20 \mathrm{~m}$, avec le «puits complet équivalent $»$ dont le rayon se tient alors aux environs du millionième de millimètre!

Par contre, dans des limites de comparaison plus raisonnables, où le jeu de la formule actuelle et des lois d'association de puits multiples permettrait de conclure à l'équivalence économique de plusieurs dispositifs de captage ou de rabattement, il conviendrait de reconnaître un avantage malheureusement difficile à chiffrer, mais surtout marqué dans les fortes perméabilités, en faveur des solutions qui procurent la plus grande surface crépinée au contact de l'aquifère.

\section{REFERENCES}

[1] Forchнemer. - Hydraulik, B. G. Teubner, Leipzig et Berlin (1930), p. 77 .

[2] Kozeny (J.). - Hydraulik, Vienne (1953), p. 422-425.

[3] Li, Benton et Bock. - Trans. Amer Geophys., Un, 35, $\mathrm{n}^{\circ} 5(1954)$, p. 805 .

[4] Boreli (M.). - Contribution à l'étude des milieux poreux. Publications scienfifiques et techniques du Ministère de l'Air, $\mathrm{n}^{\circ} 305$ (1955).

[5] Schnebeli (G.). - Limite de validité de la loi de Darcy. La Houtle Blanche, $\mathrm{n}^{\circ} 2$ (1955), p. 141-149.

[6] Tcharnyx (M.). - Article de M. Brillant. Le Génie Civil (1 ${ }^{\mathrm{er}}$ mars 1956).

\section{OUR FRONTISPICE}

\section{See page 798}

Emile YOUGUET (1871-1943)

Ingenieur Gencral des Mines

Nember of the French Acadeny of Science (1933)

Emile Jouguet zas borm on the 5th Jamary, 1871, at Bessèges (Gard), where his father, a graduate of the Ecole des Mines in Paris, was in charge of a local steel warks. His mother was the datighter of a mathematics professor at Montpallier University.

Placed fourth in his year at the Ecole Polytechnique in 1892 he entered the "Corps des Mines" and stitiled at the Ecole des Mines in Paris and was afterwards given a routine post at Bordeaut, where his natural bent for science led him to attend the lectures of Pierre $D$ whem at the Bordeaut Sciefuce Factity. Out of his contact with this fine mind grew his passion for Ener. getics, which he used in a masterly fashion, stripping it to its essentials in his efforts to perfect it.

In 1898 he took up a leaching post at the Ecole des Mines, first in Saint-Etienne and then in Paris. In 1919 he began teaching Mechanics at the Ecole Polytechnique, first as an assistant to $P$. Painlevé and then as a full professor. Retiving in 1942 he died shortly afterwards of a scrims illness. His untimely loss, in his intellectual prime, robbed French Mechanics of its diadem ara the sorry plight of France at the time provides no excuse for the inadequate homage done at the time to this faultless servan of his contry and magnificent French scientist, twho was as outstanding an engineer as a teacher and thinker.

The bulk of Emile Jouguet's work was in Mechanics, the fun damcutal natare of which he took to be dominated by Thermo dywamics. His principal folds of study were applied mechanics, contimots solid and finid media mechanics and chemical mecha nics and his work, wath covers the first forty years of this century, produced concepts both original and sound, new results and frutitul methods. It may be noted in passing and the fac will repay refection, that his theory of shock waves and com bustion in explosives, for certain aspects of which he guve the credit to Chapman and Crussard, is nozuaday more widely ktoorem and applied in the United States than in France.

For Emile Jouguet flud mechanics proper fell rather within the province of theaching than of essential research. Althotigh he published little on this subject we should note his fundancintal studies on the exeptions to d'Alembert's paradox and their phy sical significance, on similitude in fuid dyutuics and lastly the energy dissibating properties and measurchent of head loss as defined by the classical hydranlics theories of his time. In con. Hection with these particular problems he always took an active inferest in the work of the Societe Hydrotechutiqte de France. Althongh his work and preoccupations did not bring him into very clase contact with aviation, at this time in its early stages he possessed a very sure insight into the fublue directions wonich the tremendous development of the associated disciplines requir ed by this fledgling science would takc and it atas at his personal instigation that the present anthor quas led, from 1921 onteards Prandil and the famons Gottingen school, at the time almost inknown in France.

The problems of finid mechanics to which he made a major Contribution, zohtch contintes and to a certain cxtent resembles that of Hugontot between 1875 ond 1880 , concern shock wave theory and the combined effects of thermal conductivity aud vis. cosity in shock waves.

It is not generally known, for instance, but nevertheless two that it was Emile Jougutet who, half a century ago, foresat that future supersonic flow theory twould, for certaitt results, coinctdo with Newton's daring concept of a compressible fitid, first advanced over three centuries ago.

$$
\text { Matbrice ROY }
$$

Mance ROY, Minos. Member of the French Academy of Science. Professor at the Ecole Polytechnique. 